\title{
Effect of pre-miRNA-1658 gene polymorphism on chicken growth and carcass traits
}

\author{
Jianzhou Shi ${ }^{1}$ and Guirong Sun ${ }^{2, *}$
}

\author{
* Corresponding Author: Guirong Sun \\ Tel: +86-18736612881, \\ E-mail: jinbing0809@163.com
}

'Institute of Agricultural and Engineering, Nanyang Normal University, Nanyang, Henan 473061, China

${ }^{2}$ College of Livestock Husbandry and Veterinary

Engineering, Henan Agricultural University,

Zhengzhou 450002, China

Submitted Apr 21, 2016; Revised May 30, 2016; Accepted Aug 4, 2016
Objective: Polymorphisms occurring in the precursor region of microRNAs (miRNAs) affect the target gene and alter the biogenesis of miRNAs, resulting in phenotypic variation. The purpose of the study was to investigate the genetic effects of rs16681031 (C>G) mutation in the precursor region of gga-miR-1658 on the economic traits of the Gushi-Anka chicken F2 resource population.

Methods: To explore the effect of miR-1658 polymorphisms on chicken economic traits, the SNP was genotyped by MassArray matrix-assisted laser desorption/ionization-time of flight mass spectrometry. The association between the SNP and chicken body size, growth and carcass traits was determined by linear mixed models.

Results: The SNP was not only significantly associated with body weight at the age of 6, 8, 10, 12 weeks, respectively, but also with the breadth of the chicken chest, body slanting length and pelvic breadth at 4 weeks, chest depth at 8 weeks of age, and body slanting length at 12 weeks $(\mathrm{p}<0.05)$, respectively.

Conclusion: Our data serve as a useful resource for further analysis of miRNA function, and represent a molecular genetic basis for poultry breeding.

Keywords: Carcass Traits; Growth Traits; MassArray Analysis; Polymorphism; Pre-miRNA-1658

\section{INTRODUCTION}

MicroRNAs (miRNAs) are a novel class of highly conserved, small 21- to 25-nucleotide (nt) non-coding RNA molecules, which modulate the gene expression at the post-transcriptional stage by specifically interacting with target mRNAs in eukaryotes [1,2]. These single-stranded RNAs play a major role in post-transcriptional gene regulation in a sequence specific manner [3]. Growing evidence suggests that miRNAs are involved in a broad range of biological processes, including cell differentiation, cell proliferation $[4,5]$, oocyte maturation and ovarian follicular development [6,7], metabolism and apoptosis [8,9], stem cell maintenance, embryonic development [10,11], hematopoiesis [12], fat deposition [13,14], and skeletal muscle growth [15] as well as in transposon stability and tumorigenesis via regulation of gene expression $[2,16]$.

Single nucleotide polymorphisms (SNPs) represent the most abundant form of DNA variation in animal genome [17]. Common SNPs of miRNA precursors interfere with miRNA function by altering miRNA expression or maturation, resulting in phenotypic variation, including disease susceptibility. Emerging evidences supported that SNP in miRNA target sites or miRNA genes may contribute to change economic traits in domestic animals. For instance, a research reported that a SNP in the $m i R-27 a$ gene was associated with litter size in pigs [18]. SNPs in porcine microRNA miR-1 significantly altered the expression in the level of $m i R-1$, and associated with muscle fibers type compositions [19]. Bartz et al [20] reported that polymorphism of the porcine $m i R-30 d$ was associated with adipose tissue accumulation, its fatty acid profile 
and the malic enzyme 1 (ME 1) gene expression. An et al [17] reported that single-nucleotide polymorphisms g.151435C $>\mathrm{T}$ and g.173057T $>C$ in prolactin receptor $(P R L R)$ gene regulated by $b t a-m i R-302 a$ were associated with litter size in goats. Gga$m i R N A-133 a$ and $g g a-m i R-1 a$ were reported to be related to skeletal muscle, whereas gga-miR-122 was adipose-related miRNA in chickens [21]. A research promulgated that chicken gga-miR-202* was involved in regulating the development of embryonic gonad in chickens [22]. An SNP in pre-miR-27a (rs895819) affects miRNA expression, serves as a risk factor for breast cancer and reduces breast cancer risk in younger Chinese population through genetic variants [23]. The SNP rs2910164 in pre-miR-146 affects the coronary artery disease incidence through a common variant [24]. Increasing evidence suggests that SNP in miRNA target sites or miRNA genes are likely to contribute to economic traits in domestic animals. Meat quality traits, body size and growth are the most important economic traits in poultry genetics and breeding. Lower feed conversion and production is an important concern of the poultry industry. In this study, we identified an SNP located in the precursor region of the gga-miR-1658, and analyzed its association with carcass traits and body weight of chickens in a Gushi-Anka F2 resource population. Our data identified the potential role of $m i R-1658$ in growth development.

\section{MATERIALS AND METHODS}

\section{Study population}

A total of 860 chickens from a Gushi-Anka F2 resource population previously generated by the Henan Innovative Engineering Research Center of Poultry Germplasm Resource [25] were used for the study. The F2 generation resulted from a cross between the native Chinese Gushi chicken breed, which represents an excellent meat and slow-growing variety, and Anka broilers, which are large-bodied and fast-growing broilers. The GushiAnka F2 resource population created from 4 cross families and 2 reciprocal cross families, was composed of 42 grandparents ( 2 roosters and 24 hens from Gushi chicken, 4 roosters and 12 hens from Anka chicken), $70 \mathrm{~F} 1$ parental chickens and 860 F2 chickens produced from two hatches 2 weeks apart by intercrossing $7 \mathrm{~F} 1$ cockerels with $63 \mathrm{~F} 1$ hens. One-day-old F2 chickens were raised in the top 3 levels of 4-level-coops with 50 chickens per coop, providing $392 \mathrm{~cm}^{2} /$ chick. At 56 days of age, chickens were moved to triple stair-step coops in groups of 3 per coop, providing $448 \mathrm{~cm}^{2}$ per chick. All the birds were managed in cages under similar environment, including free access to water and food ad libitum until 12 weeks, according to the Chinese National Research Council [26]. All the chickens were slaughtered under similar conditions at the age of 84 days. In this study, all the experimental animals used were handled according to the national and institutional guidelines of animal care in research.

\section{Traits}

The body weight of the birds was weighed individually, measured and recorded every 2 weeks from birth to 12 weeks of slaughter, starting with hatching, 2, 4, 6, 8, 10, and 12 weeks later. The body size traits mainly included shank length (SL), shank girth (SG), chest depth $(\mathrm{CD})$, chest breadth $(\mathrm{CB})$, breast bone length (BBL), pectoral angle (PA), body slanting length (BSL), and pelvis breadth $(\mathrm{PB})$ at different growth stages. The carcass traits such as semi-evisceration weight (SEW), the carcass weight excluding reproductive organs, spleen, pancreas, gallbladder, crop, esophagus, intestine, and trachea), evisceration weight (EW, SEW excluding the abdominal fat, gizzard, liver, head, proventriculus, feet, and heart), breast muscle weight (BMW), leg muscle weight (LMW), and carcass weight (CW) and so forth were accurately measured after slaughtering.

\section{Polymorphism detection}

The genomic DNA samples in 860 F2 chickens were extracted from the jugular vein blood anticoagulated with ethylenediaminetetraacetic acid using standard phenol-chloroform methods in molecular biology [27]. In accordance with the criteria for matrix-assisted laser desorption/ionization-time of flight mass spectrometry (MALDI-TOF MS) genotyping, the genomic DNA was adjusted to accurate concentrations and stored at $-80^{\circ} \mathrm{C}$ for further utilization. One hundred DNA samples from the F2 resource population individuals were selected randomly, and equal amounts of similar working concentrations of each DNA sample were used to construct a DNA pool. The miR-1658 gene sequence, which is located in intron 13 of the guanine monophosphate synthase 201 (GMPS-201) host gene on chromosome 9, and chicken pre-miR-1658 sequence (GenBank accession no: MI0007392) were obtained from the miRBase database version 19.0 (http://www.mirbase.org). A pair of polymerase chain reaction (PCR) sequencing primers (forward: $5^{\prime}$ - GAGA TCTTGGATTACAAG - $3^{\prime}$ and reverse: 5'- GTTCGTAGA TACAAAGTAAG - $3^{\prime}$ ) was designed according to the genomic location (Accession no: NC_006096.3) to scan SNPs in gga$m i R-1658$. The primers were used to amplify the molecular fragment containing the $g g a-m i R-1658$ gene precursor region. The DNA pool was sequenced by Sangon Biotech Co. Ltd. (Shanghai, China), to detect polymorphisms. All the genomic DNA samples were sent to a reputed diagnostic company to identify the SNP using the MassArray MALDI-TOF MS assay.

\section{Genotyping}

The rs16681031 SNP occurring in miR-1658 (+60 bp C>G) in the F2 chickens was genotyped using MassArray-iPLEX GOLD System (Sequenom Inc., USA). The SNP genotyping was accomplished with a pair of sequence amplification primers and a single-base extension primer designed using Sequenom software Assay Design 3.1 version. The first PCR primer sequence of pre-mir-1658 SNP was 5'- ACGTTGGATGTTCTGCCATAC 
CAGTGTGTG -3'; the second PCR primer sequence was 5'ACGTTGGATGACTCCTCCAGCTGCTGCTTC $-3^{\prime}$, and the single-base extension primer was 5'- CATCAACACCAACCCA $-3^{\prime}$. The SNP of pre-mir-1658 was genotyped using Sequenom MALDI-TOF mass spectrometry according to the manufacturer's instructions [28]. Related genotyping data including the peak area and call rate were obtained. Alleles were assigned automatically, using the software package provided by the manufacturer.

\section{The prediction of pre-miR-1658 secondary structure}

According to the rs16681031 SNP with $C>G$ alleles in the precursor region of miRNA-1658 in chickens, the most stable secondary structure of pre-mir-1658 with the lowest free energy was calculated by M-fold [29]. The absolute difference in the free energy for pre-mir-1658 with different alleles was used as the parameter for the assessment of the impact on the secondary structure of pre-miR-1658.

\section{Statistical analysis}

The SPSS20.0 Statistics software (SPSS for Windows, Standard version 20.0; SPSS, USA) was used for the association analysis of the correlation between pre-miRNA-1658 SNP and the economic traits of the F2 chickens. The relationship between polymorphism and the F2 population-related economic traits was analyzed using a procedure with two linear mixed models. Model I was used to evaluate growth traits and model II was used to calculate the carcass traits. Considering the effect of body weight differences on carcass traits, the model II carcass weight was used as a covariate to investigate its effect on carcass traits. In case of significant effect of genotype, the Bonferroni test was carried out for multiple comparisons of the genotypes. Less than $0.05 \mathrm{p}$ value was considered considered statistically significant. In the analysis, the two mixed linear models were as follows:

Model I: $Y_{\mathrm{ijkl}}=\mu+\mathrm{G}_{\mathrm{i}}+\mathrm{S}_{\mathrm{j}}+\mathrm{H}_{\mathrm{k}}+\mathrm{f}_{1}+\mathrm{e}_{\mathrm{ijk} \mathrm{kl}}$

$$
\text { Model II: } Y_{\mathrm{ijkl}}=\mu+\mathrm{G}_{\mathrm{i}}+\mathrm{S}_{\mathrm{j}}+\mathrm{H}_{\mathrm{k}}+\mathrm{f}_{\mathrm{l}}+\mathrm{b}\left(\mathrm{W}_{\mathrm{ijklm}}-\mathrm{W}\right)+\mathrm{e}_{\mathrm{ijk} k \mathrm{~m}}
$$

Where $Y_{\mathrm{ijkl}}$ represents the observed value; $\mu$ the overall mean, $G_{i}$ the fixed effect of genotype ( $i=3$ : CC, CG, GG); $f_{1}$ the random effect of family $(l=7)$; $S_{j}$ fixed effect of sex $(j=2), H_{k}$ the fixed effect of hatch $(k=2), e_{i j k l m}$ the random error, $b$ the regression coefficient of carcass weight; $\mathrm{W}_{\mathrm{ijk} \text { km }}$ the individual carcass weight; and $\mathrm{W}$ the average carcass weight. Less than $0.05 \mathrm{p}$ value was considered statistically significant.

\section{RESULTS}

\section{Identification and genotyping of miR-1658 SNP}

The polymorphism was verified by PCR product sequencing results compared with the published chicken genome sequence, showing the occurrence of a $\mathrm{C}>\mathrm{G}$ mutation at +60 bp of pre$m i R-1658$ in the F2 resource population, in Figure 1. The MALDI-TOF mass spectrograms of variant genotypes CC, GC, and GG are displayed in Figure 2, respectively. The allele frequencies of the SNP in the gga-miR-1658 gene of the F2 resource population were 0.746 for $\mathrm{C}$ and 0.254 for $\mathrm{G}$, and the genotype frequencies were $0.525,0.441$, and 0.034 for $\mathrm{CC}, \mathrm{GC}$, and GG, respectively.

\section{Second structure alterations of the $m i R-1658$ precursor}

The result showed that the $\mathrm{C}>\mathrm{G}$ variation located in the seed region of pre-miR-1658 could introduce a base-pairing mismatch, resulted in generation of a new RNA bulge in the predicted second structure, with the altering of the free energy (Figure 3).

\section{Association of $m i R-1658 \mathrm{SNP}$ with performance traits} To investigate the role of +60 bp $C>G$ pre-miR-1658 polymorphism on poultry phenotypic traits, the SNP was correlated with the body weight at different times (at $0,2,4,6,8,10$, and 12 weeks), and carcass traits at 12 weeks and presented in Table

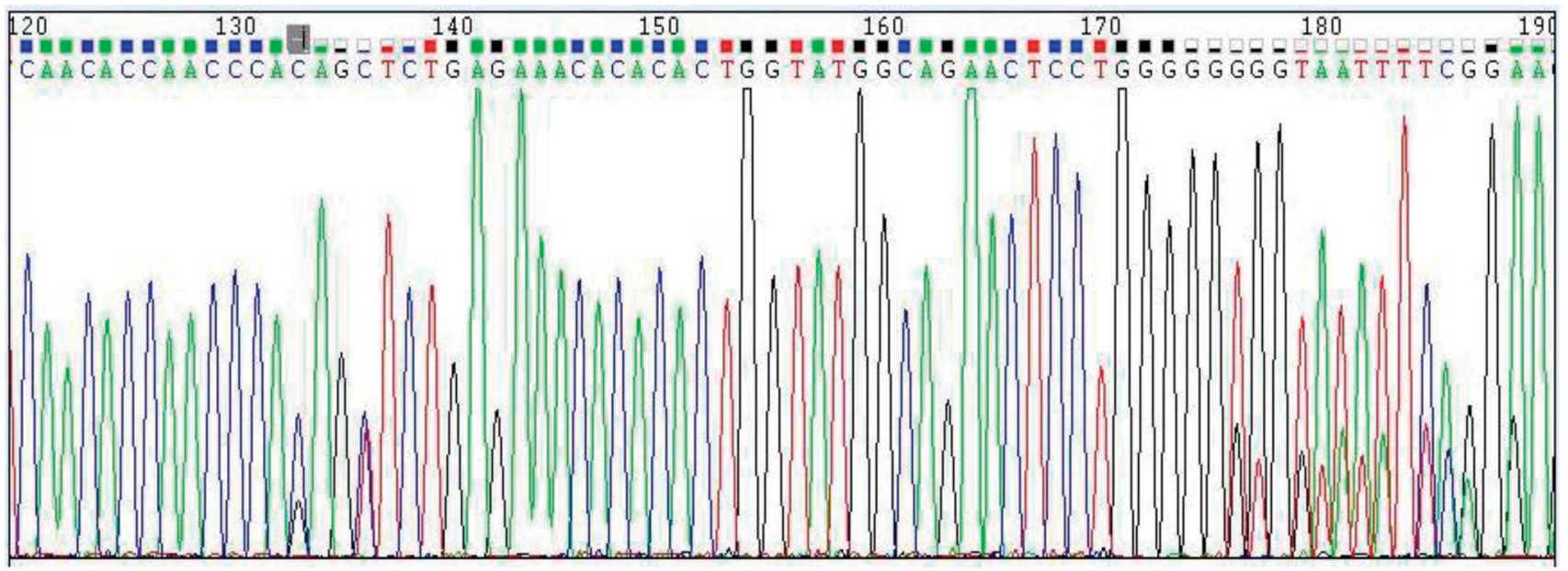

Figure 1. Polymerase chain reaction analysis of miR-1658. The base in the shadow represents the mutation site. 


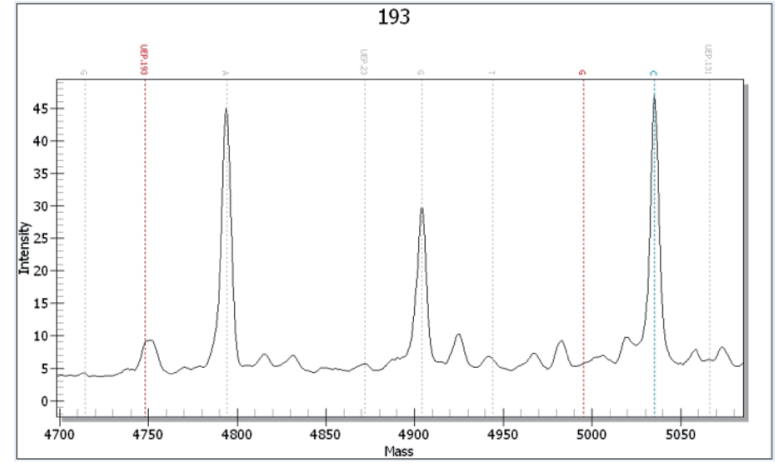

CC genotype

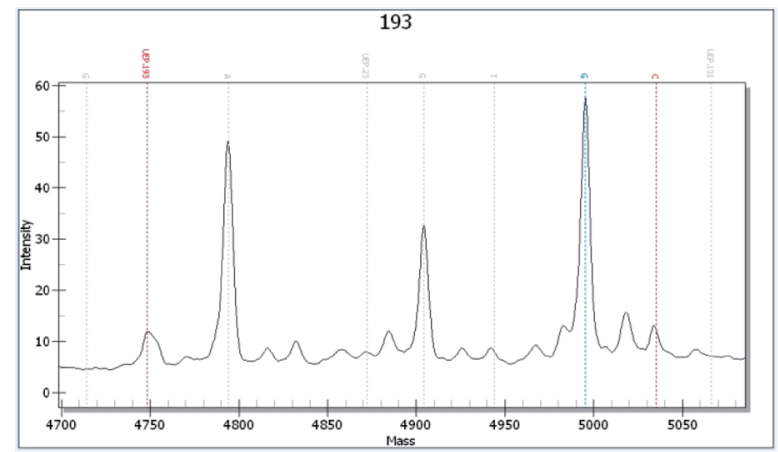

GG genotype

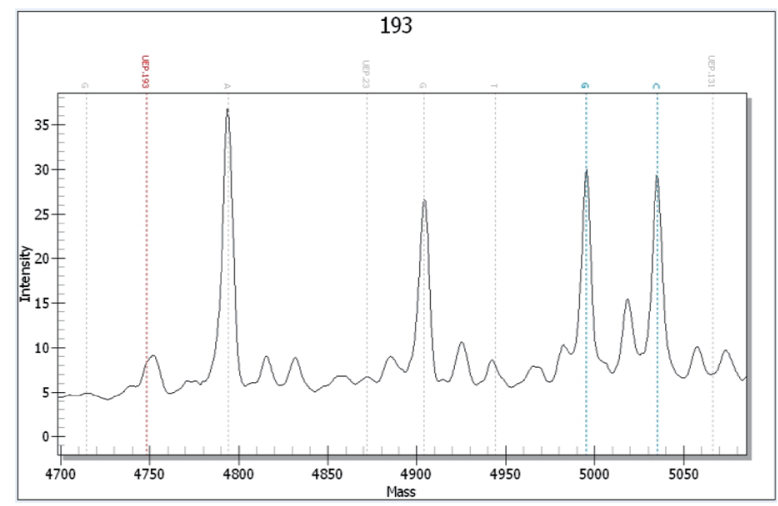

GC genotype

Figure 2. Mass spectrogram of $C C, G G$, and $\mathrm{GC}$ polymorphism of $\mathrm{C}>\mathrm{G}$ in premir-1658.

1. The $m i R-1658 \mathrm{SNP}(+60 \mathrm{bp} \mathrm{C}>\mathrm{G})$ had a significant $(\mathrm{p}<0.05)$ effect on body weight at the age of $6,8,10$, and 12 weeks in the Gushi-Anka F2 resource population of chickens. These mutations indirectly affect the body weight at different developmental stages and carcass traits of the chickens. Association analysis of rs16681031 genotype with chicken body size is depicted in Table 2. At the age of 4 weeks, the SNP was significantly associated with the chicken $\mathrm{CB}, \mathrm{BSL}$, and $\mathrm{PB}(\mathrm{p}<0.05)$. Chickens with the GG genotype were the largest on $\mathrm{CB}, \mathrm{BSL}$, and $\mathrm{PB}$. Individuals with GG genotype were lower than the CC genotype on BSL. The SNP was significantly associated with the chicken CD at 8 weeks $(\mathrm{p}<0.05)$. At 12 weeks, the $\mathrm{C}>\mathrm{G}$ polymorphism was
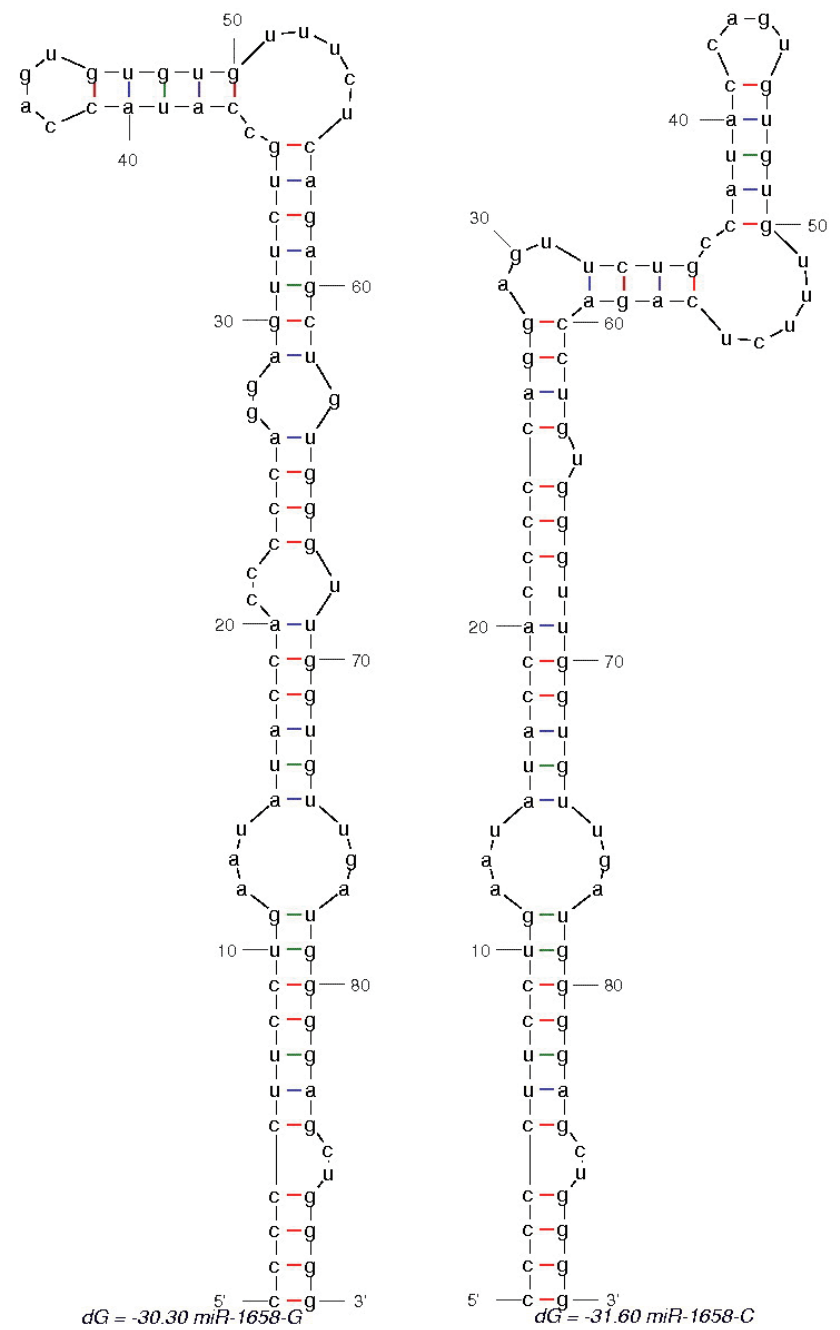

Figure 3. The predicted secondary structure of the pre-gga-miR-1658 with different alleles.

significantly linked to BSL ( $\mathrm{p}<0.05)$. Chickens with CC genotype showed a greater 8-week CD and 12-week BSL, compared with the GC genotype.

\section{DISCUSSION}

Studies have shown that a common variant in pre-miRNA is associated with disease or cancer risk, and is probably associated with phenotypic variation, including disease susceptibility, including a few miRNA SNPs serving as genetic markers of disease in clinical trials [30,31]. However, few reports investigated the polymorphism within the pre-miRNA genes for commercially viable traits in animals. Wang et al [14] reported that three novel melanocortin-4 receptor (MC4R) SNPs were associated with growth traits in $\mathrm{Hu}$ sheep and East Friesian $\times \mathrm{Hu}$ crossbred sheep. The association analysis between three SNPs and body traits indicated that two of the SNPs were associated with body weight, except for g.306 G>A mutation. All the three loci were 
Table 1. Association of miR-1658(+60 C>G) polymorphism with chicken growth and carcass traits

\begin{tabular}{|c|c|c|c|c|}
\hline \multirow{2}{*}{ Traits $^{1)}$} & \multicolumn{3}{|c|}{ SNP genotype (Mean \pm SE) } & \multirow{2}{*}{$p$-value } \\
\hline & $\mathrm{CC}$ & GC & GG & \\
\hline SEW (g) & $1,127.82 \pm 179.11$ & $1,084.77 \pm 187.30$ & $1,049.69 \pm 150.50$ & 0.667 \\
\hline BMW (g) & $72.73 \pm 14.52$ & $69.36 \pm 16.73$ & $70.33 \pm 14.51$ & 0.342 \\
\hline LMW (g) & $100.99 \pm 19.51$ & $96.79 \pm 20.21$ & $92.08 \pm 14.08$ & 0.910 \\
\hline CW (g) & $1,242.11 \pm 191.13$ & $1,194.65 \pm 199.27$ & $1,152.61 \pm 154.93$ & 0.359 \\
\hline 4BW (g) & $326.45 \pm 46.64$ & $318.00 \pm 49.61$ & $317.72 \pm 40.28$ & 0.110 \\
\hline $6 \mathrm{BW}(\mathrm{g})$ & $574.14 \pm 104.97^{\mathrm{a}}$ & $552.88 \pm 111.15^{b}$ & $555.59 \pm 71.89^{b}$ & 0.021 \\
\hline $8 B W(g)$ & $833.28 \pm 137.70^{a}$ & $801.11 \pm 146.21^{b}$ & $789.44 \pm 95.95^{b}$ & 0.048 \\
\hline 10BW $(g)$ & $1,138.75 \pm 172.65^{\mathrm{a}}$ & $1,093.89 \pm 189.47^{b}$ & $1,070.75 \pm 116.22^{b}$ & 0.026 \\
\hline $12 \mathrm{BW}(\mathrm{g})$ & $1,381.97 \pm 210.25^{a}$ & $1,326.36 \pm 228.63^{b}$ & $1,286.75 \pm 173.39^{b}$ & 0.026 \\
\hline
\end{tabular}

SNP, single nucleotide polymorphism; SE, standard error; SEW, semi-evisceration weight; EW, evisceration weight; BMW, breast-muscle weight; LMW, leg muscle weight; CW, carcass weight.

1) $0,2,4,6,8,10,12 \mathrm{BW}$, body weight at the age of 0 day, $2,4,6,8,10$, and 12 weeks, respectively.

a,b Indicate values within a row with no common superscript differing significantly $(p<0.05)$.

significantly associated with the body size [14]. The study involving Nanyang cattle showed that SNPs within bovine hepatocyte growth factor $(H G F)$ gene were significantly associated with

Table 2. Association of miR-1658 ( +60 C > G) polymorphism with chicken body size

\begin{tabular}{|c|c|c|c|c|}
\hline \multirow{2}{*}{ Traits $^{1)}$} & \multicolumn{3}{|c|}{ SNP genotype (Mean \pm SE) } & \multirow{2}{*}{$\mathrm{p}$-value } \\
\hline & CC & GC & GG & \\
\hline OSL (cm) & $2.58 \pm 0.02$ & $2.56 \pm 0.02$ & $2.57 \pm 0.06$ & 0.885 \\
\hline 4SL (cm) & $5.68 \pm 0.11$ & $5.45 \pm 0.12$ & $5.28 \pm 0.36$ & 0.313 \\
\hline $8 \mathrm{SL}(\mathrm{cm})$ & $8.25 \pm 0.12$ & $7.82 \pm 0.14$ & $8.06 \pm 0.40$ & 0.136 \\
\hline $12 S L(c m)$ & $9.72 \pm 0.13$ & $9.28 \pm 0.14$ & $9.69 \pm 0.41$ & 0.26 \\
\hline $4 S G(\mathrm{~cm})$ & $2.77 \pm 0.04$ & $2.71 \pm 0.04$ & $2.88 \pm 0.13$ & 0.185 \\
\hline $8 S G(\mathrm{~cm})$ & $3.52 \pm 0.04$ & $3.39 \pm 0.05$ & $3.50 \pm 0.14$ & 0.126 \\
\hline $125 \mathrm{sG}(\mathrm{cm})$ & $3.99 \pm 0.05$ & $3.83 \pm 0.06$ & $3.85 \pm 0.16$ & 0.199 \\
\hline $4 C D(\mathrm{~cm})$ & $4.95 \pm 0.09$ & $4.95 \pm 0.10$ & $4.78 \pm 0.31$ & 0.371 \\
\hline $8 C D(\mathrm{~cm})$ & $7.01 \pm 0.15^{b}$ & $6.30 \pm 0.17^{\mathrm{a}}$ & $5.83 \pm 0.49^{\mathrm{a}}$ & 0.033 \\
\hline $12 \mathrm{CD}(\mathrm{cm})$ & $8.09 \pm 0.14$ & $8.15 \pm 0.15$ & $8.63 \pm 0.45$ & 0.377 \\
\hline $4 C B(\mathrm{~cm})$ & $4.22 \pm 0.07^{\mathrm{a}}$ & $3.89 \pm 0.08^{b}$ & $4.25 \pm 0.24^{\mathrm{ab}}$ & 0.007 \\
\hline $8 \mathrm{CB}(\mathrm{cm})$ & $5.72 \pm 0.10$ & $5.67 \pm 0.11$ & $5.63 \pm 0.32$ & 0.963 \\
\hline $12 \mathrm{CB}(\mathrm{cm})$ & $6.62 \pm 0.13$ & $6.25 \pm 0.14$ & $6.13 \pm 0.41$ & 0.146 \\
\hline 4BBL (cm) & $6.36 \pm 0.10$ & $6.32 \pm 0.11$ & $6.35 \pm 0.32$ & 0.309 \\
\hline $8 \mathrm{BBL}(\mathrm{cm})$ & $8.89 \pm 0.17$ & $8.85 \pm 0.18$ & $8.93 \pm 0.54$ & 0.283 \\
\hline $12 \mathrm{BBL}(\mathrm{cm})$ & $11.27 \pm 0.11$ & $10.95 \pm 0.12$ & $11.10 \pm 0.34$ & 0.131 \\
\hline $4 \mathrm{PA}\left({ }^{\circ}\right)$ & $73.89 \pm 0.65$ & $75.08 \pm 0.71$ & $75.25 \pm 2.11$ & 0.207 \\
\hline $8 \mathrm{PA}\left({ }^{\circ}\right)$ & $77.58 \pm 0.74$ & $77.09 \pm 0.81$ & $77.00 \pm 2.40$ & 0.805 \\
\hline $12 \mathrm{PA}\left({ }^{\circ}\right)$ & $78.70 \pm 0.81$ & $79.23 \pm 0.88$ & $80.75 \pm 2.62$ & 0.710 \\
\hline 4BSL (cm) & $11.60 \pm 0.12^{a}$ & $10.98 \pm 0.13^{b}$ & $11.50 \pm 0.39^{\mathrm{ab}}$ & 0.000 \\
\hline $8 B S L(c m)$ & $16.47 \pm 0.19$ & $16.16 \pm 0.21$ & $16.53 \pm 0.61$ & 0.521 \\
\hline 12BSL (cm) & $20.47 \pm 0.16^{\mathrm{a}}$ & $19.58 \pm 0.17^{b}$ & $20.23 \pm 0.51^{\mathrm{ab}}$ & 0.004 \\
\hline $4 P B(\mathrm{~cm})$ & $5.28 \pm 0.08^{\mathrm{a}}$ & $5.03 \pm 0.08^{b}$ & $5.38 \pm 0.25^{\mathrm{ab}}$ & 0.028 \\
\hline $8 \mathrm{~PB}(\mathrm{~cm})$ & $7.06 \pm 0.12$ & $6.82 \pm 0.13$ & $6.88 \pm 0.38$ & 0.374 \\
\hline $12 \mathrm{~PB}(\mathrm{~cm})$ & $8.88 \pm 0.15$ & $8.81 \pm 0.17$ & $8.90 \pm 0.50$ & 0.682 \\
\hline
\end{tabular}

SNP, single nucleotide polymorphism; SE, standard error; SL, shank length; SG, shank girth; $C D$, chest depth; $C B$, chest breadth; $B B L$, breast bone length; $P A$, pectoral angle; $B S L$, body slanting length; $P B$, pelvis breadth.

${ }^{1)} 0,4,8$, and 12 represent weeks.

Means with different superscripts in the line suggest significant differences: lower case denotes $p<0.05$. growth traits [32]. Yu et al [33] reported that a G/T polymorphism (ss52050737) in exon 11 was significantly associated with the total number of ova and the number of transferable embryos by examining polymorphisms in luteinizing hormone/choriogonadotropin receptor (LHCGR) and the genotypes associated with superovulation traits in 127 Chinese Holstein heifers. SNPs in miRNAs may alter miRNA expression or maturation, leading to miRNA regulatory function. Increasing evidence suggests that SNPs in miRNAs and their target sites alter miRNA function. The miRNA mutations leading to aberrant miRNA-mediated gene regulation may modulate the transcription and stability of pri-miRNA and pre-miRNAs, resulting in phenotypic variation [34]. Therefore, it is essential to investigate the SNPs in miRNA and study their association with economic traits in domestic animals. Zorc et al [35] found that some miRNAs had highly polymorphic seed regions, pre-miRNA gga-mir-1658 had one SNP within the mature sequence (gga-miR-1658) and two SNPs within the minor miR sequence ( $\left.g g a-m i R-1658^{*}\right)$, in addition, in chicken $g g a-m i R-1658$ with seven quantitative trait locus. Jevsinek et al [36] reported that polymorphisms residing within the miRNA seed region of chicken gga-mir-1658 were selected for experimental validation. The rs 16681031 and rs16681032 SNP is located in seed regions of chicken $\mathrm{gga}$-mir1658-3p which resides in the intron 13 of miRNA host guanine monphosphate synthetase (GMPS) gene, the rs16681033 SNP is located in seed regions of gga-mir-1658-5p which resides in the intron 13 of GMPS gene [36]. Geng et al [37] reported that the rs 16681031 SNP located in seed regions of gga-mir-1658* in 6 chicken populations 180 individuals. The results represented that genotype frequencies of the gga-mir-1658* C $>$ G has significant differences in Beijing Fatty Chicken and other chicken breeds [37]. Geng et al [38] investigated that polymorphism of pre-microRNA-1658 gene in chicken. Additionally, the seed zone mutation could affect the stability of gga-mir-1658 gene 
precursor secondary structure and the selection of target genes, which might be the important functional site having potential phenotype effect [38]. This study has reported the pre-miR-1658 SNP of an F2 resource population for the first time. In this study, we identified a SNP located in the seed zone of the miR-1658 and investigated its association with body weight and body indexes traits at different developmental stages in chickens. The association analysis between the SNP genotype in the gga-miR-1658 precursor region and growth and carcass traits showed a significant correlation between genotype and economic traits. Our work suggested that a point mutation occurring in miR-1658 was significantly associated with body weight and body indexes traits in chickens. The gga-mir-1658 gene seed region mutation could affect the selection of target genes and the stability of precursor secondary structure in chickens, which might be the important functional site having phenotype effect.

Further, rs16681031 was associated with body weight at 6, 8,10 , and 12 weeks of age and also with $8 \mathrm{CD}, 4 \mathrm{CB}, 4 \mathrm{BSL}, 12 \mathrm{BSL}$, and $4 \mathrm{~PB}$. The relationship between the genotype and carcass traits was not significant, which was unexpected considering our previous hypothesis. Our results revealed that the genotype CC showed the heaviest body weight compared with chickens containing genotypes GC and GG, except birth weight. At birth, chickens showing the CC pattern had a lower body weight than those exhibiting the GC genotype, but greater than the GG. Statistical analysis showed that chickens with GG genotype showed the greatest body size at 4,8 , and 12 weeks (4SG, 4CB, $4 \mathrm{PA}, 4 \mathrm{~PB}, 8 \mathrm{BBL}, 8 \mathrm{BSL}, 12 \mathrm{PA}$, and 12PB). The SNP in the $g g a-$ $m i R-1658$ precursor region showed an important effect on the commercial traits of the F2 resource population of chickens. The result indicated that the SNP may alter the processing efficiency of pre-miRNA to mature miRNA, thereby altering the function. Therefore, pre-miR-1658 might represent a potential candidate gene controlling growth traits. The probability of a positive association with the body weight was presumed for the presence of $\mathrm{G}$ allele in the $\mathrm{SNP}+60 \mathrm{C}>\mathrm{G}$ of the $g g a-m i R-1658$ gene precursor in chickens. The mechanism of phenotypic variation attributed to pre-miRNA-1658 polymorphism needs further study.

In conclusion, we identified and established the rs16681031 $(+60 \mathrm{C}>\mathrm{G})$ SNP within the pre-miR-1658 gene in the F2 generation of the Gushi-Anka chicken population. The association analysis revealed a significant impact of pre-miR-1658 SNP on growth and carcass traits at different developmental stages. These results enable early artificial selection and breeding of poultry. These findings provide insight into the role of biomarkers and molecular genetics of poultry breeding. Further investigation into pre-miR-1658 genetics in different chicken populations is needed.

\section{CONFLICT OF INTEREST}

We certify that there is no conflict of interest with any financial organization regarding the material discussed in the manuscript.

\section{ACKNOWLEDGMENTS}

The work was supported by the National Natural Science Foundation of China (No.31201795), the Postdoctoral Science Foundation of China (2013M531675), the Colleges and Universities Key Scientific Research Project in Henan (16B230006), and the High-level Personnel Scientific Research Foundation of Nanyang Normal University (2013022).

\section{REFERENCES}

1. Aigner A. MicroRNAs (miRNAs) in cancer invasion and metastasis: therapeutic approaches based on metastasis-related miRNAs. J Mol Med (Berl) 2011;89:445-57.

2. Li H, Sun GR, Lv SJ, et al. Association study of polymorphisms inside the miR-1657 seed region with chicken growth and meat traits. Br Poult Sci 2012;53:770-6.

3. Sand M, Gambichler T, Sand D, et al. MicroRNAs and the skin: tiny players in the body's largest organ. J Dermatol Sci 2009;53:16975

4. Shi XE, Li YF, Jia L, et al. MicroRNA-199a-5p affects porcine preadipocyte proliferation and differentiation. Int J Mol Sci 2014;15:852638.

5. Zhang J, Zhao H, Gao Y, Zhang W. Secretory miRNAs as novel cancer biomarkers. Biochim Biophys Acta 2012;1826:32-43.

6. Fiedler SD, Carletti MZ, Hong X, Christenson LK. Hormonal regulation of MicroRNA expression in periovulatory mouse mural granulosa cells. Biol Reprod 2008;79:1030-7.

7. Mahdipour M, van Tol HT, Stout TA, Roelen BA. Validating reference microRNAs for normalizing qRT-PCR data in bovine oocytes and preimplantation embryos. BMC Dev Biol 2015;15:25.

8. Chen Y, Verfaillie CM. MicroRNAs: the fine modulators of liver development and function. Liver Int 2014;34:976-90.

9. Kim VN, Han J, Siomi MC. Biogenesis of small RNAs in animals. Nat Rev Mol Cell Biol 2009;10:126-39.

10. Li H, Wang S, Yan F et al. Effect of polymorphism within miRNA-1606 gene on growth and carcass traits in chicken. Gene 2015;566:8-12.

11. Wang X, Gu Z, Jiang H. MicroRNAs in farm animals. Animal 2013; 7:1567-75.

12. Lazare SS, Wojtowicz EE, Bystrykh LV, de Haan G. microRNAs in hematopoiesis. Exp Cell Res 2014;329:234-8.

13. Lin S, Li H, Mu H, et al. Let-7b regulates the expression of the growth hormone receptor gene in deletion-type dwarf chickens. BMC Genomics 2012;13:306.

14. Wang H, Xiao S, Wang M, et al. In silico identification of conserved microRNAs and their targets in bovine fat tissue. Gene 2015;559: 119-28.

15. Luo W, Nie Q, Zhang X. MicroRNAs involved in skeletal muscle differentiation. J Genet Genomics 2013;40:107-16. 
16. Ambros V. The function of animal MicroRNAs. Nature 2004;431: 350-5.

17. An X, Hou J, Gao T, et al. Single-nucleotide polymorphisms g.151435C $>\mathrm{T}$ and g.173057T >C in PRLR gene regulated by bta-miR-302a are associated with litter size in goats. Theriogenology 2015;83: 1477-83.

18. Lei B, Gao S, Luo LF, et al. A SNP in the miR-27a gene is associated with litter size in pigs. Mol Biol Rep 2011;38:3725-9.

19. Hong JS, Noh SH, Lee JS, et al. Effects of polymorphisms in the porcine microRNA miR-1 locus on muscle fiber type composition and miR-1 expression. Gene 2012;506:211-6.

20. Bartz M, Koscianska E, Szczerbal I, et al. Polymorphism of the porcine miR-30d is associated with adipose tissue accumulation, its fatty acid profile and the ME1 gene expression. Livest Sci 2015;182:54-7.

21. Wang X, Yu J, Zhang Y, Gong D, Gu Z. Identification and characterization of microRNA from chicken adipose tissue and skeletal muscle. Poult Sci 2012;91:139-49.

22. Bannister SC, Tizard ML, Doran TJ, Sinclair AH, Smith CA. Sexually dimorphic microRNA expression during chicken embryonic gonadal development. Biol Reprod 2009;81:165-76.

23. Zhang N, Huo Q, Wang X, et al. A genetic variant in pre-miR-27a is associated with a reduced breast cancer risk in younger Chinese population. Gene 2013;529:125-30.

24. Xiong XD, Cho M, Cai XP, et al. A common variant in pre-miR-146 is associated with coronary artery disease risk and its mature miRNA expression. Mutat Res 2014;761:15-20.

25. Han R, Wei Y, Kang X, et al. Novel SNPs in the PRDM16 gene and their associations with performance traits in chickens. Mol Biol Rep 2012;39:3153-60.

26. Han RL, Li ZJ, Li MJ, et al. Novel 9-bp indel in visfatin gene and its associations with chicken growth. Br Poult Sci 2011;52:52-7.

27. Sambrook J. Russell DW. Molecular cloning: a laboratory manual (3rd Edition). New York: Cold Spring Harbor Laboratory Press;
2001.

28. Oeth P, Beaulieu M, Park C, et al. PLEX $^{\mathrm{TM}}$ assay: increased plexing efficiency and flexibility for MassARRAY ${ }^{\circledR}$ system through single base primer extension with mass-modified terminators. SEQUENOM ${ }^{\mathbb{R}}$ Application Note; 2005.

29. Zuker M. Mfold web server for nucleic acid folding and hybridization prediction. Nucleic Acids Res 2003; 31:3406-15.

30. Jazdzewski K, Murray EL, Franssila K, et al. Common SNP in premiR-146a decreases mature miR expression and predisposes to papillary thyroid carcinoma. Proc Natl Acad Sci USA 2008;105: 7269-74.

31. Zhang J, Ying ZZ, Tang ZL, Long LQ, Li K. MicroRNA-148a promotes myogenic differentiation by targeting the ROCK1 gene. J Biol Chem 2012;287:21093-101.

32. Cai H, Lan X, Li A, et al. SNPs of bovine HGF gene and their association with growth traits in Nanyang cattle. Res Vet Sci 2013;95:483-8.

33. Yu Y, Pang Y, Zhao H, et al. Association of a missense mutation in the luteinizing hormone/choriogonadotropin receptor gene (LHCGR) with superovulation traits in Chinese Holstein heifers. J Anim Sci Biotechnol 2012;3:35.

34. Georges M, Coppieters W, Charlier C. Polymorphic miRNA-mediated gene regulation: contribution to phenotypic variation and disease. Curr Opin Genet Dev 2007;17:166-76.

35. Zorc M, Skok DJ, Godnic I, et al. Catalog of MicroRNA seed polymorphisms in vertebrates. PLOS ONE 2012;7: e30737.

36. Jevsinek S, Godnic I, Zorc M, et al. Genome-wide in silico screening for microRNA genetic variability in livestock species. Anim Genet 2013;44:669-77.

37. Geng L, Zhang C, Li Y, et al. The chicken GGA-Mir-1658* Gene: seed region polymorphisms, frequency distribution and putative targets. J Anim Vet Adv 2011;10:1187-93.

38. Geng L, Zhang C, Zhao S, et al. Polymorphism of pre-microRNA-1658 gene in chicken. Sci Agric Sin 2015;48:3919-30. 